\title{
Acupoint Application Therapeutic Mechanism for Novel Coronavirus Pneumonia: A Network Pharmacology Analysis
}

\section{Shan Liu}

Guangzhou University of Chinese Medicine

chi zhang

Guangzhou university of chinese medicine https://orcid.org/0000-0001-8826-8498

\section{Zexin Zhang}

Guangzhou University of Chinese Medicine

\section{Wei Guo}

Guangzhou University of Chinese Medicine Jiangnan Xia

Guangzhou University of Chinese Medicine Jing Li

Guangzhou University of Chinese Medicine

\section{Zhijie Wang ( $\square$ wang15244616809@126.com )}

Center for Evidence-based Chinese Medicine, Beijing University of Chinese Medicine, Beijing, 100029, China https://orcid.org/0000-0002-9361-6570

\section{Research}

Keywords: acupoint application therapy, Asarum, Ephedra, mechanism, network pharmacology, novel coronavirus pneumonia (COVID-19)

Posted Date: December 18th, 2020

DOI: https://doi.org/10.21203/rs.3.rs-129282/v1

License: (a) (i) This work is licensed under a Creative Commons Attribution 4.0 International License. Read Full License 


\section{Abstract}

Background and objective: Acupoint application (AA) therapy, a traditional Chinese medicine external treatment method, is recommended as a complementary Chinese medicine therapy for treating new coronavirus pneumonia (COVID-19), and can help improve curative effects, promote patient recovery, and improve immunity to prevent disease, but the mechanism is not clear. We sought to explore the key targets and mechanisms of Ephedra and Asarum, the main components used in AA, in the treatment of COVID-19 in this study.

Methods: The active compounds and related targets of Ephedra and Asarum used in AA were screened by searching four databases. COVID-19-related target genes were identified from three disease databases, and the key targets of treating COVID-19 were obtained by Venn diagram using Cytoscape 3.2.1 software, gene ontology (GO) enrichment analysis, and Kyoto Encyclopedia of Genes and Genomes (KEGG) enrichment analysis were used to explore possible mechanisms and pathways. The verification of the binding force between the compounds and target genes was completed by performing molecular docking with Autodock and pymol software.

Results: Twenty-four active components of AA corresponded to 252 targets, with 713 target genes related to COVID-19, and 56 key genes were selected. GO analysis results showed that biological processes mainly included the inflammatory and immune responses, and cell components were mainly the plasma membrane and nucleus, with molecular functions including protein binding and identical protein binding. The top pathways included Immune System, Cytokine Signaling in Immune System, Adaptive Immune System, Signaling by Interleukins, Innate Immune System, and Signaling by GPCR.

Conclusion: The results of this study preliminarily verified that Ephedra and Asarum used in AA therapy could treat COVID-19 via multiple compounds and multi-pathways, which provided a basis for clinical application and further research.

\section{Introduction}

Since December 2019, a new type of coronavirus pneumonia (COVID-19) has spread from China around the world, attracting global attention. As of October 15, 2020, there have been 38,202,956 confirmed cases of COVID-19, including 1,087,069 deaths worldwide, reported to the World Health Organization [1].

COVID-19 is an acute infectious disease caused by severe acute respiratory syndrome coronavirus type 2 (SARS-CoV-2). The main transmission route is through air and direct contact, with a possibility of aerosol transmission and fecal-oral transmission [2]. The most common symptoms include fever, cough, and fatigue, while other symptoms include headache, hemoptysis, diarrhea, and difficulty in breathing [3].

At present, there is no specific medicine for COVID-19, and treatment mainly includes nutritional interventions, immunoenhancers, coronaviral protease inhibitors, antiviral agents, oxygen therapy, circulatory support, and traditional Chinese medicine (TCM) [4-6]; the latter has been used to treat epidemic diseases for thousands of years [7]. 
The "National Pneumonia Diagnosis and Treatment Plan for Novel Coronavirus Infection (7th Version)" stated that COVID-19 belongs to the category of "plague" [8]. Prior to its release, the National Health Commission of China and The State Administration of Traditional Chinese Medicine had recommended TCM treatments for COVID-19, since the 3rd version of this plan, as it showed good clinical effects. TCMs, such as Qingfei Paidu decoction, Gancao Ganjiang decoction, Shegan Mahuang decoction, and Qingfei Touxie Fuzheng recipe, have been reported to be beneficial in treating COVID-19. A meta-analysis including seven original studies, comprising 732 adults in total, showed that TCM helped to improve treatment outcomes in COVID-19 cases [9]. Lianhua Qingwen formula significantly relieves clinical symptoms in patients with fever, weakness, cough, and could reduce the course of COVID-19 [10]. Moreover, the combination of Shufeng Jiedu capsule and Arbidol was better than Arbidol alone in COVID-19 treatment, and could significantly shorten the symptom improvement time [11].

In addition to oral medicines, acupoint application (AA), acupuncture, moxibustion, fumigation, and other external treatments have also been used for treating COVID-19 [12]. AA has been recommended as an auxiliary therapy for COVID-19 in hospitalization and in personal protection. It is easy to implement, noninvasive, and effective $[13,14]$. AA applies TCM, such as pills, powders, or ointments, into acupoints, to prevent and treat diseases by using the dual effects of drug transdermal absorption and meridian effects [15]. It is widely used for treating vascular and respiratory diseases, including pneumonia, chronic obstructive pulmonary disease, bronchial asthma, chronic cough, and other diseases [16-18].

Different Chinese herbal medicines have been used in AA for treating diverse diseases, and Ephedra, Asarum, mustard seeds, cinnamon, bitter almonds, and Corydalis rhizome are used more frequently in AA for respiratory disease. Ephedra and Asarum are the Chinese herbal medicine pair most commonly used for COVID-19 $[12,19,20]$. Oral decoctions, such as the Huashi Baidu formula and Qingfei Paidu Decoction, which also contain Ephedra and Asarum, are recommended by clinical guidelines for the treatment of mild, common, and severe COVID-19 [21-24]. The ancient classics of Chinese medicine showed that these two Chinese herbal medicines have good curative effects in relieving cough and panting, and reducing fever. In modern pharmacological research, Ephedra has been shown to have effects against sweating, antipyretic and antiviral effects, and to relieve cough and asthma $[25,26]$. Asarum has been shown to have good antiinfluenza virus activity in vitro, and could play a protective role by reducing the viral load and inflammatory damage in lung tissue, and by regulating the expression of inflammatory cytokines [27]. Like Ephedra, Asarum relieves cough and fever and regulates immunity [28]. We assumed that AA may treat COVID-19 through the above mechanisms, and thus selected Ephedra and Asarum for detailed mechanistic exploration in the present study.

Although there have been reports about the chemical components, pharmacological mechanisms, and clinical use of AA, there has been no systematic, overall analysis of its components, targets, pathways, and mechanism of interaction. TCM has complex characteristics of unity and integrity, and is based on the theory of "holistic concept" and "dialectical treatment." Network pharmacology can be used to explore the correlation between drugs and diseases in an integrated and systematic manner, and considers drug and target interactions, consistent with basic TCM characteristics. Therefore, network pharmacology is a new approach for systematically revealing the role of TCM in body network regulation. It uses computer 
simulations and various databases to screen targets for drug molecules, to predict their signaling pathways and mechanism of action, and the software is used to visualize the compound-target-pathway network, of which the key nodes are screened by topological parameter analysis. It partly addresses the limitation of "single component-single target point-single pathway" in TCM research [29].

In, this study, we used network pharmacology to obtain the active compounds and targets of Ephedra and Asarum, as a key TCM pair used in AA for COVID-19. We established related networks to analyze the effective components and mechanisms by which this AA treatment affects COVID-19. The flowchart of the network pharmacology approach is shown in Fig. 1.

\section{Materials And Methods}

\section{Screening of active components of AA}

We used the Traditional Chinese Medicine Systems Pharmacology Database Analysis Platform (TCMSP http://lsp.nwu.edu.cn /index.php), Traditional Chinese Medicines Integrated Database (TCMID http://119.3.41.228:8000/tcmid/), bioinformatics analysis tool for molecular mechanisms of TCM (http://bionet.ncpsb.org/batman-tcm/), and PubChem (https://pubchem.ncbi.nlm.nih.gov/) to identify the main constituents of Ephedra and Asarum. To screen for more active TCM compounds, this study combined half-life $(\mathrm{HL})$ and drug similarity $(\mathrm{DL})$ to predict the pharmacokinetics of the compounds. DL reflected the similarity between specific functional groups in the compound and known drugs, and DL evaluation helped to improve the accuracy of compound screening. $\mathrm{HL}$ is a pharmacokinetic parameter, reflecting the time it takes for the concentration of a drug to degrade by half in the body. It was mainly used as a time measurement of compound efficacy. In this study, $\mathrm{DL} \geq 0.18$, and $H L \geq 4$ were set as standards to screen potential active components.

\section{Screening TCM putative targets}

We continued to use the above-mentioned database as a platform to search for the key compounds in Ephedra and Asarum to find the corresponding protein targets. Uniport (https://www.uniprot.org/) was searched to obtain the standard target names.

\section{Screening of COVID-19-related targets}

We used "2019-nCoV," "COVID-19," or "Novel Coronavirus Pneumonia" as keywords in Genecards (https://www.genecards.org/), Therapeutic Target Database (TTD, http://bidd.nus.edu.sg/group/cjttd/), and Online Mendelian Inheritance in Man (OMIM, https://www.omim.org/) to search for COVID-19-related targets. These were then imported into an EXCEL table for standardization for subsequent statistical analysis.

\section{Protein-protein interaction network construction of AA- and COVID-19-related target genes}


The STRING database (https://string-db.org/) was used to construct a protein-protein interaction (PPI) network of putative AA- and COVID-19-related target genes, and the following settings were used:

Textmining, Experiments, and Databases. The minimum required interaction score was 0.7 , which indicated high confidence. Finally, we disabled the structure previews inside network bubbles and we hid disconnected nodes in the network. Cytoscape software and GraphPad Prism software were used to construct the PPI barplot, which showed the significant target genes.

\section{Identification of candidate genes and construction of TCM component-target network}

To identify common active targets, we mapped the identified targets of Ephedra and Asarum and the COVID-19 targets by using Bioinformatics \& Evolutionary Genomics

(http://bioinformatics.psb.ugent.be/webtools/Venn/) and constructed a Venn diagram to show putative targets of these Chinese medicines for COVID-19. We then obtained the PPI network using the STRING database (https://string-db.org/) to reveal the mechanism by which these AA components act against COVID-19. Cytoscape 3.2.1 software was used to visualize the network diagram and its plugins as CMODE, clustermaker2, and a network analyzer was used to select the active target according to the degree value. Then, we obtained module clusters and analyzed their mutual relations. To explain the mechanism of the active compounds in Ephedra and Asarum against COVID-19, this study constructed a visual "componenttarget" network in Cytoscape 3.2.1. In this network, compounds and targets are represented by nodes, and the interaction between two nodes is represented by edges.

\section{Gene Ontology and Kyoto Encyclopedia of Genes and Genomes pathway enrichment}

To analyze the biological and signal pathway conduction processes in the actions of AA against COVID-19, and to clarify the mechanism of drug action, the targets in the network were imported into the Comparative Toxicogenomics Database (CTD, http://ctdbase .org/) and the DAVID database (https://david.ncifcrf.gov/) for gene ontology (GO) analysis (including biological processes [BP], molecular functions [MF], and cellular components [CC]) and the Kyoto Encyclopedia of Genes and Genomes (KEGG) pathway enrichment. "ggplot2" packages from R 3.5.3 software was used to draw related charts. Finally, the relationship between target genes and pathways was presented as a target-pathway network diagram in Cytoscape. To verify the results, we imported the target genes into R 3.6.1 software and used the "clusterprofiler" and "pathview" packages to obtain the specific mechanism of gene enrichment in signaling pathways.

\section{Molecular docking}

To verify the reliability of the findings, we used Autodock and pymol software to dock constituents and target genes and displayed docking results in terms of binding energy. The lower this energy, the stronger the binding ability of the components and target genes.

\section{Results}


Through database searches, 653 and 365 components of Ephedra and Asarum, respectively, were selected as highly active compounds with $\mathrm{DL} \geq 0.18$, and $\mathrm{HL} \geq 4$ as standards. After we deleted compounds without a target, 24 potential active compounds with good ADME properties, including quercetin, kaempferol, luteolin, naringenin, beta-sitosterol, and stigmasterol, were identified for further analysis (Table 1).

\section{Putative targets of herbs used in AA and COVID-19-related target genes}

A total of 647 potential human targets were identified for the above 24 compounds (Asarum: 171; Ephedra: 476). After deleting the duplicated targets, 252 targets were finally obtained. Additionally, after deleting the duplicated genes among 736 disease-related genes obtained from the three disease databases, 713 COVID19-related target genes were identified .

\section{PPI network of putative AA and COVID-19-related target genes}

The PPI network of putative AA-related target genes (Figure 2A) contained 236 nodes and 1889 edges and that of COVID-19-related target genes (Figure 2B) contained 352 nodes and 2588 edges. We then used Cytoscape software and GraphPad Prism software to construct the PPI barplot, which showed the target genes significant to both AA and COVID-19 (Figure 2C and 2D).

To obtain AA targets that interact with the disease, we used the Bioinformatics \& Evolutionary Genomics platform to match potential AA target genes with disease-related genes and drew a Venn diagram (Figure 3). Finally, 56 putative AA targets that interacted with disease-related targets were identified.

\section{PPI network analysis of AA action against COVID-19}

We imported the 56 key targets into the STRING database to generate a PPI network (Figure 4A) (settings: species Homo sapiens, confidence level >0.7, STRING database confidence level high > 0.7; medium > 0.4; and low >0.15).

The PPI results were imported into Cytoscape software, and clustermaker2 and Network analyzer plugins were used to generate a subnetwork (Figure 4B) containing 53 nodes and 388 edges. The network radius was two, and the average number of neighbors was 14.642. The size of the circle and the font indicate the degree value, and different colors indicate the number of adjacent points connected. The top10 degrees were IL6 $($ degree $=34)$, TNF $($ degree = 34), MAPK1 (degree = 31), TP53 $($ degree $=30)$, MAPK8 $($ degree = 30), IL1B (degree = 28), RELA (degree = 27), CASP3 (degree = 25), STAT1 (degree = 23), MAPK3 (degree = 23), and IL10 (degree $=22)$.

Subsequently, the MCODE plug-in in Cytoscape was used for further cluster analysis, and two module clusters in the network were generated (Table 2 and Figure 5). Module 1 had the highest node, edge number, and score values. This may be the main model by which the targets exert their effects; this module included IL6, TNF, PTGS2, IL1B, MAPK1, MAPK3, MAPK8, MAPK14, among others. This cluster consisted of 
14 component nodes and 74 edges, the clustering confidence was 0.887 , and the average number of neighbors was 11. STAT1 (marked in yellow in Figure 5) was the seed of the cluster; this target might thus play an important role in connecting other nodes in the PPI network.

\section{Component-target network analysis}

We used Cytoscape 3.2.1 software to construct a network and analyze the 24 compounds and 252 target genes identified (Figure 6). The network contained 275 nodes (nodes) and 582 edges (edges); the red square represents the compound, the blue circle represents the target protein, and the edges represent the relationship. The network density was 0.015 , the distance was 5 , and the median of 24 candidate components was 10 degrees, indicating multiple target genes. The top-three effective components were quercetin, kaempferol, and luteolin, which had 154, 65, and 58 target genes, respectively. This network verified that Ephedra and Asarum had a therapeutic effect through multi-component, multi-target, integrated regulation.

\section{GO function enrichment analysis}

We imported the 56 target genes into the DAVID database and used R 3.5.3 software to perform GO functional enrichment analysis. The 10 most significant BPs in Figure 7A included an inflammatory response, immune response, positive regulation of transcription from RNA polymerase II promoter, response to drug, positive regulation of transcription, DNA-template, positive and negative regulation of apoptotic process, apoptotic process, and cellular response to lipopolysaccharide. The top-10 CCs in Figure 7B included the plasma membrane, nucleus, cytoplasm, cytosol, nucleoplasm, extracellular space, extracellular exosome, integral component of plasma membrane, extracellular region, and mitochondrion. The top-10 MFs in Figure 7C included protein binding, identical protein binding, enzyme binding, protein homodimerization activity, ATP binding, protein heterodimerization activity, DNA binding, transcription factor activity, sequence-specific DNA binding, protein kinase binding, and zinc ion binding.

\section{KEGG pathway enrichment analysis}

As shown in Figure 8A, the herbs affected COVID-19 through multiple signaling pathways; we selected 20 related pathways with corrected $\mathrm{P}$ values $\leq 0.01$ and gene frequency $>0.5 \%$. In this network, the color represents the $p$ value, and the length represents the number of genes. The top pathways, indicating the key pharmacological mechanisms of the AA herbs against COVID-19, included the Immune System, Cytokine Signaling in the Immune System, Adaptive Immune System, Signaling by Interleukins, Innate Immune System, Signaling by GPCR, Hemostasis, HTLV-I infection, Signal Transduction囚Pathways in cancer, PI3KAkt signaling pathway.

To understand the relationship between protein targets and signal pathways, we selected 10 pathways with higher degree values to establish a target-pathway network diagram in Figure 8B. The network had 59 nodes and 217 edges. The green squares represent the pathway and the yellow circles represent the targets. 
In the subsequent pathway verification, we found that the IL-17, Influenza A, and TNF signaling pathway were consistent with our previous pathway enrichment analysis. The red marks in Figure $8 \mathrm{C}-8 \mathrm{E}$ represent potential target genes related to $A A$.

\section{Molecular docking}

Quercetin and kaempferol, selected from the above methods were considered to be the main candidate compounds, and IL-6, TNF, and STAT1 were considered to be the core targets. Molecular docking revealed that two TCM compounds bound to TNF with the lowest binding energy (i.e., $-7.8 \mathrm{kcal} / \mathrm{mol}$, and the others were -7.6 [quercetin and IL-6], -7.3 [quercetin and STAT1], -7.2 [kaempferol and STAT1], and -6.9 [kaempferol and IL-6]) (Table 3 and Figure 9).

\section{Discussion}

AA is an important part of the external Chinese medicine treatment. For COVID-19, AA can stimulate body surface points, reconcile qi and blood, stimulate meridians, and adjust the body's immune function [30]. Some clinical studies have shown that AA can reduce adverse drug reactions in patients with COVID-19 [31]. Therefore, this study used network pharmacology methods to identify the active compounds of Ephedra and Asarum, the main components used in AA, to obtain their key targets for COVID-19, Then, we established network diagrams, and analyzed the related biological processes and signal pathways. In this way, using modern pharmacological principles, the role of Ephedra and Asarum in treating COVID-19 were systematically discovered to embody the pharmacological theory of TCM as being "multi-target" and "multi-disease" [32].

This study identified 24 active components and 56 active targets for these two herbs. The components quercetin, kaempferol, $\beta$-sitosterol, stigmasterol, luteolin, and naringin were surrounded by considerable targets, and can be considered as the main active components. An animal experiment has shown that quercetin has a protective effect against lipopolysaccharide-induced acute lung injury in rats, which may be related to reduced release of pro-inflammatory factors [33]. Quercetin could also inhibit elevated expression of cysteine protease 3 (CASP3) protein in virus-infected cells and antagonize virus-induced cell apoptosis $[34,35]$. $\beta$-sitosterol and stigmasterol are phytosterols, mainly found in seeds, beans, and fruits, and have anti-inflammatory, anti-tumor, and hypolipidemic effects [36]. Animal studies have shown that $\beta$-sitosterol increases the activity of superoxide dismutase and reduces inflammation [37]. Liz et al. found that $\beta$ sitosterol exerts anti-inflammatory effects by promoting cellular calcium uptake, and inhibiting IL-1 $\beta$ and TNF-a [38]. Kaempferol also scavenges oxygen free radicals and suppresses the expression of inflammatory factors, such as IL-1 $\beta$, IL-6, and TNF, by downregulating the MAKP signaling pathway, thereby reducing inflammation [39]. Luteolin and naringin also have antiviral and anti-inflammatory effects [40]. Thus, component screening and analysis of component-target networks revealed that many components in Ephedra and Asarum exert anti-viral, anti-inflammatory, and immune regulatory effects. Our findings fully validated the multi-component and multi-channel network characteristics of TCM in treating COVID-19, and was consistent with the basic theories of Chinese medicine, such as integrated concepts, syndrome differentiation, and compatibility rule [41]. 
By analyzing the PPI network diagram and performing GO enrichment analysis, we identified 56 key targets involved in particular BPs, such as the immune response, inflammatory response, cell proliferation, and apoptosis responses, which were closely related to the occurrence and progression of COVID-19. Targets with higher degree values included IL6, TNF, IL1B, CASP3, STAT1, MAPK3, and IL10. In COVID-19 patients, the levels of inflammatory factors, such as IL1B, IL10, and TNF, are significantly increased and correlate positively with the disease severity [42]. IL6, IL1B, and IL10 are lymphokines that interact with white blood cells or immune cells. These factors transmit information, regulate the proliferation and differentiation of immune cells, and play an important role in the inflammatory response. IL-6 has a very high clinical application value. For early infections, it could assist in the diagnosis, and judging the prognosis and response to treatment [43]. Clinical studies have shown that AA could control patients' symptoms, improve lung function, and reduce levels of inflammatory factors, including IL-6 [44]. TNF- $\alpha$ is the initiating factor of the cytokine network, inducing the release of other cytokines, such as IL- 6 and IL- $1 \beta$, tissue damage, and the cytokine storm $[45,46]$. In the analysis of module clusters, we found that STAT1 is a central point within the target network, playing an important role in connecting other targets and pathways. STAT1 is a key factor in the IFN- $\alpha / \beta$ signaling pathway which mediates production of various downstream inflammatory factors [47]. STAT1-knockout mice are significantly more susceptible to infection by various pathogens (viruses, bacteria, parasites, and fungi) [48]. Other targets, such as MAPK1, MAPK8, RELA, and MAPK3, form part of the RAS pathway, which could directly or indirectly regulating the expression level of ACE2, thereby inhibiting SARS-CoV-2 and reducing the cytokine storm [49]. Taken together, our findings indicate that the mechanism of action of Ephedra and Asarum against COVID-19 involved BPs, such as immune response and inflammatory response regulation, and anti-viral effects.

Finally, through KEGG pathway enrichment, we showed that most of the main pathways in TCM target enrichment were significantly related to inflammation, cytokines, and immunity; these confirmed our PPI screening results. Therefore, Ephedra and Asarum used in AA might activate interactive pathways, such as the interleukin, TNF, and Influenza A signaling pathways, through targets such as IL6, TNF, IL1B, CASP3, STAT1, MAPK3, and IL10. In this way, AA could regulate the immune response and cell proliferation and apoptosis, thereby exerting anti-inflammatory, anti-viral, and other pharmacological effects, which could be effective against COVID-19.

\section{Conclusions}

In this study, network pharmacology methods were used to make preliminary predictions about the components, active targets, and mechanism of action of the main components of Asarum and Ephedra used in AA. This provides a theoretical basis for subsequent experimental research and drug discovery. However, we selected only the two most common components of AA for analysis. In addition, our research could not control the influence of factors such as point selection, drug dosage, and drug production area. These factors and specific mechanisms need to be investigated further in future studies.

\section{Abbreviations}


AA

Acupoint application; ADME:absorption, distribution, metabolism, and excretion; BP:biological processes; CC:cellular components; COVID-19:New coronavirus pneumonia; CTD:Comparative Toxicogenomics Database; DL:drug-likeness; GO:Gene Ontology; HL:half-life; IL-6:Interleukin 6; KEGG:Kyoto Encyclopedia of Genes and Genomes; MF:molecular functions; OMIM:Online Mendelian Inheritance in Man; STAT1:signal transducer and activator of transcription 1; TCM:traditional Chinese medicine; TCMID:Traditional Chinese Medicine Integrated Database; TCMSP:traditional Chinese medicine system pharmacology analysis platform; TNF:Tumor necrosis factor; TTD:Therapeutic Target Database.

\section{Declarations}

\section{Authors' contributions}

SL, CZ, JL, WG and ZJW conceived and designed the study. SL, CZ and JNX drafted the manuscript. CZ and ZXZ collected the data. CZ and ZXZ performed the data analysis. ZJW, and WG provided advice during the study and manuscript preparation. All authors have read and approved the final manuscript.

\section{Ethics approval and consent to participate}

Not applicable

\section{Consent for publication}

Not applicable

\section{Availability of data and material}

The datasets used and analyzed during the current study are available from the corresponding author upon reasonable request.

\section{Competing interests}

The authors declare that they have no competing interests.

\section{Funding}

This study was supported by the 2020 Hunan University of Traditional Chinese Medicine, Chinese Medicine Prevention and Treatment of Novel Coronavirus Pneumonia Scientific Research Project plan (2020XGXM08).

\section{Author information}

Shan Liu, Email: liushan @gzucm.edu.cn.

Zhijie Wang, Email: wang15244616809@126.com. 


\section{Footnotes}

Shan Liu, Chi Zhang, Zexin Zhang and Wei Guo are the co-first authors.

\section{Acknowledgements}

Not applicable

\section{References}

1. [Internet] World health Organization. WHO Coronavirus Disease (COVID-19) Dashboard. Available online: https://covid19.who.int/.

2. Holshue ML, DeBolt C, Lindquist S, Lofy KH, Wiesman J, Bruce H, et al. First case of 2019 novel coronavirus in the United States. N Engl J Med. 2020; doi: 10.1056/NEJMoa200119.

3. Huang C, Wang Y, Li X, Ren L, Zhao J, Hu Y, et al. Clinical features of patients infected with 2019 novel coronavirus in Wuhan, China. Lancet. 2020;395:497-506.

4. Zhang L, Liu Y. Potential interventions for novel coronavirus in China: A systematic review. J Med Virol. 2020;92:479-90.

5. Jin YH, Cai L, Cheng ZS, Cheng H, Deng T, Fan YP, et al. A rapid advice guideline for the diagnosis and treatment of 2019 novel coronavirus (2019-nCoV) infected pneumonia (standard version). Mil Med Res. 2020;7:4.

6. Yu F, Du L, Ojcius DM, Pan C, Jiang S. Measures for diagnosing and treating infections by a novel coronavirus responsible for a pneumonia outbreak originating in Wuhan, China. Microbes Infect. 2020;22:74-9.

7. Su B, Liu QQ. On the relationship between influenza, warm disease and Shanhan. Journal of Traditional Chinese Medicine. 2019;60:1249-51.

8. Publicity Department of the People's Republic of China. Press conference of the joint prevention and control mechanism of state council on Feb 17, 2020. http://www.nhc.gov.cn/xcs/fkdt/202002/f12a62d10c2a48c6895cedf2faea6e1f.shtml(accessed Feb 23, 2020; in Chinese).

9. Fan AY, Gu S, Alemi SF; Research Group for Evidence-based Chinese Medicine. Chinese herbal medicine for COVID-19: Current evidence with systematic review and meta-analysis. J Integr Med. 2020;31:S2095-4964(20)30078-9. doi: 10.1016/j.joim.2020.07.008.

10. Cheng DZ, Li Y. Clinical effectiveness and case analysis in 54 NCP patients treated with Lianhuaqingwen granules. World Chin Med. 2020;15:150-4.

11. Qu XK, Hao SL, Ma JH, Wei GY, Song KY, Tang C, et al. Observation on clinical effect of Shufeng Jiedu Capsule combined with Arbidol Hydrochloride Capsule in treatment of COVID-19. Chin Tradit Herbal Drugs. 2020;51:1167-70.

12. Zhou DH, Chang H. Discussion on the application value of external treatment of Chinese medicine in COVID-19. Acta Chinese Medicine:1-5[2020-05-04]. 
http://kns.cnki.net/kcms/detail/41.1411.r.20200302.1809.002.html.

13. Li XH, Zhong F, Cao Y. Preliminary analysis of rules of medicinal composition in acupoint application for bronchial asthma based on data mining. Shanghai Journal of Acupuncture and Moxibustion. 2019;38:806-11.

14. Li HM, Yang ZJ, Xie M, Zhao Y, Zuo XH. Analysis of novel coronavirus pneumonia (COVID-19) close contacts with Chinese medicine preventive measures. Asia-Pacific Traditional Medicine, 2020;16:1-4.

15. Wang Y, Hong LZ, Lei B. A survey of treating allergic rhinitis with application of acupoint. J Clin Acupunct Moxibustion 2010;26:68-70.

16. Jin LH, Guo SC, Zeng JJ. Clinical study on point application with Chinese herbal medicine for acute exacerbation of chronic obstructive pulmonary disease. Journal of New Chinese Medicine. 2019;51:300-3.

17. Guo SJ, Shi LQ, Ji K, Wang LM, Song H, Ma JL, et al. Epidemiological characteristics of acupoint application therapy of winter disease cured in summer on treatment of respiratory diseases. Journal of Liaoning University of Traditional Chinese Medicine 2019;21:77-80.

18. Li N, Zhang BJ, Niu FH, Yu GZ, Dong Q. Research progress of acupoint application of traditional Chinese medicine in the treatment of respiratory diseases in children. China Journal of Traditional Chinese Medicine and Pharmacy. 2019;34:5331-3.

19. Luo H, Zhao ZM, Xin YJ. Exploration into acupoint application as adjuvant therapy in treating COVID19. Henan Traditional Chinese Medicine. 2020;40:831-4.

20. Yang JJ. Study on the selection of acupoints and medications of acupuncture treatment for chronic pulmonary winter disease. (Master Thesis) Shanxi University of Traditional Chinese Medicine, Taiyuan, China, 2018.

21. Yang R, Liu H, Bai C, Wang Y, Zhang X, Guo R, et al. Chemical composition and pharmacological mechanism of Qingfei Paidu Decoction and Ma Xing Shi Gan Decoction against Coronavirus Disease 2019 (COVID-19): In silico and experimental study. Pharmacol Res. 2020;157:104820.

22. Gao K, Song YP, Chen H, Zhao LT, Ma L. Therapeutic efficacy of Qingfei Paidu decoction combined with antiviral drugs in the treatment of corona virus disease 2019: A protocol for systematic review and meta analysis. Medicine. 2020;99:e20489.

23. Tao Q, Du J, Li X, Zeng J, Tan B, Xu J, et al. Network pharmacology and molecular docking analysis on molecular targets and mechanisms of Huashi Baidu formula in the treatment of COVID-19. Drug Dev Ind Pharm. 2020;46:1345-53.

24. Han L, Wang Y, Hu K, Tang Z, Song X. The therapeutic efficacy of Huashi Baidu Formula combined with antiviral drugs in the treatment of COVID-19: A protocol for systematic review and meta-analysis. Medicine. 2020;99:e22715.

25. Li JL, Fang L, Zhang YQ, Wang X, Zhang QD. Research progress on chemical constituents and pharmacological activities of Ephedra. Modern Chinese Medicine. 2012;14:21-7.

26. Mantani N, Imanishi N, Kawamata H, Terasawa K, Ochiai $\mathrm{H}$. Inhibitory effect of (+)-catechin on the growth of influenza A/PR/8 virus in MDCK cells. Planta Med. 2001;67:240-3. 
27. Yang J, Fu YP, Du BX, Yang Y, Rong R. Effect of Asarum polysaccharide in protecting H1N1 influenza virus infection in vitro and on expressions of inflammatory factors. China Journal of Chinese Materia Medica:1-9. [2020-10-29].https://doi.org/10.19540/j.cnki.cjcmm.20200929.401.

28. Wu H, Wen XY, Yan P, Shen SR, Liu XD, Lai KF. Research progress on chemical constituents and pharmacological effects of Asari radix et rhizoma. Chinese Journal of Experimental Traditional Medical Formulae:1-9. [2020-10-29]. https://doi.org/10.13422/j.cnki.syfjx.20202353.

29. Ren Y, Deng YJ, Ma HB, et al. Research progress and challenges of network pharmacology in field of traditional Chinese medicine. Chinese Traditional and Herbal Drugs. 2020;51:4789-97.

30. Wang Y, Zhang W. 82 cases of hepatogenic diarrhea treated by shugan zhixie decoction combined with acupoint application. Chinese Journal of Integrated Traditional and Western Medicine on Liver Diseases. 2017,27:115-6.

31. Lai JM, Zheng YF, Hu CY, Zeng X. Observation on curative effect of acupoint application of warming spleen and relieving diarrhea in treating new coronary pneumonia antiviral diarrhea. Zhejiang Journal of Integrated Traditional Chinese and Western Medicine. 2020;30:268-70.

32. Yu D, Ye T, Xiang Y, Shi Z, Zhang J, Lou B, et al. Quercetin inhibits epithelial-mesenchymal transition, decreases invasiveness and metastasis, and reverses IL-6 induced epithelial-mesenchymal transition, expression of MMP by inhibiting STAT3 signaling in pancreatic cancer cells. Onco Targets Ther. 2017;10:4719-29.

33. Liu SW. Protective Effects of Quercetin on Lipopolysaccharide-induced Acute Lung Injure in Rats and Effects of Nrf/ARE Signaling Pathway. (Master Thesis) Zunyi Medical University, Zunyi, China, 2019.

34. Wan QF, Wu L, Yang ML, Ma R, Liang J, Gu LG. Effect of Quercetin on Caspase-3 of A549 Cell Induced by Influenza Virus H1N1. Chinese Journal of Information on Traditional Chinese Medicine. 2011;18:424.

35. Wu W, Li R, Li X, He J, Jiang S, Liu S, et al. Quercetin as an antiviral agent inhibits Influenza A virus (IAV) entry. Viruses. 2016;8:6.

36. Geng J Z, Liang J M, Xu LL. The physiological functions of phytosterol and its development prospects. Beverage Industry 2015;5:70-3.

37. Xiao ZB, Liu XL, Cheng RQ, Jia HX, Wang XY, Kong LN, et al. Influence of $\beta$-sitosterol on gastric mucosal side effect induced by aspirin and its pharmacological functions. Chinese Journal of Experimental Traditional Medical Formulae. 2016;22:148-52.

38. Liz R, Zanatta L, dos Reis GO, Horst H, Pizzolatti MG, Silva FR , et al.Acute effect of $\beta$-sitosterol on calcium uptake mediates anti-inflammatory effect in murine activated neutrophils. J Pharm Pharmacol. 2013;65:115-22.

39. Zhang YW, Shao DY, Shi JL, Zhu J, Huang QS, Yang H. A review on biological activities of kaempferol. Chinese Bulletin of Life Sciences. 2017;29:400-5.

40. Wang ZY, Sun YZ, Qu ND, Liu BY, Fan Z, Tian JZ. Network pharmacological study on mechanism of Maxing Shigan Decoction in treatment of coronavirus disease 2019 (COVID-19). Chinese Traditional and Herbal Drugs. 2020;51:1996-2003. 
41. Zhang YQ, Li S. Progress in network pharmacology for modern research of traditional Chinese medicine. Chinese Journal of Pharmacology and Toxicology. 2015;29:883-92.

42. Rothan HA, Byrareddy SN. The epidemiology and pathogenesis of coronavirus disease (COVID-19) outbreak. J Autoimmun. 2020;109:102433.

43. Kaur S, Bansal Y, Kumar R, Bansal G. A panoramic review of IL-6:Structure, pathophysiological roles and inhibitors. Bioorg Med Chem. 2020;28:115327

44. Zhao BL. Effect of acupoint application of Chinese medicine in "Dong disease Xia Zhi" adjuvant treatment on children's asthma, lung function, IL-6 and TNF-a levels. Inner Mongolia Medical Journal. 2020;52:160-12.

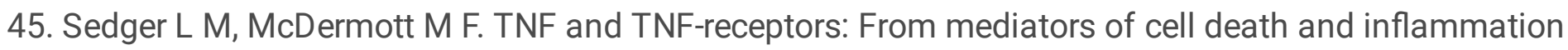
to therapeutic giants-past, present and future. Cytokine Growth Factor Rev. 2014;25:453-72.

46. Yang CQ, Zhai J, Liu YT, et al. Immune imbalance mechanism and intervention strategy in patients with coronavirus disease 2019 (COVID-19). Chinese Pharmacological Bulletin, 2020;36:445-53.

47. Dale TC, Imam AM, Kerr IM, Stark GR. Rapid activation by interferon alpha of a latent DNA-binding protein present in the cytoplasm of untreated cells. Proc Natl Acad Sci USA. 1989;86:1203-7.

48. Olbrich P, Freeman AF. STAT1 and STAT3 mutations: important lessons for clinical immunologists. Expert Rev Clin Immunol. 2018;14:1029-41.

49. Sun X, Tao JL, Xu SJ. The Molecular Mechanism of Treating COVID-19 with Huashii Baidu Formula based on Network Pharmacology. Journal of Chinese Medicinal Materials:1-7 [2020-05-05]. http://kns.cnki.net/kcms/detail/44.1286.R.20200430.1759.006.html.

\section{Tables}

Due to technical limitations, table 1-3 is only available as a download in the Supplemental Files section.

\section{Figures}




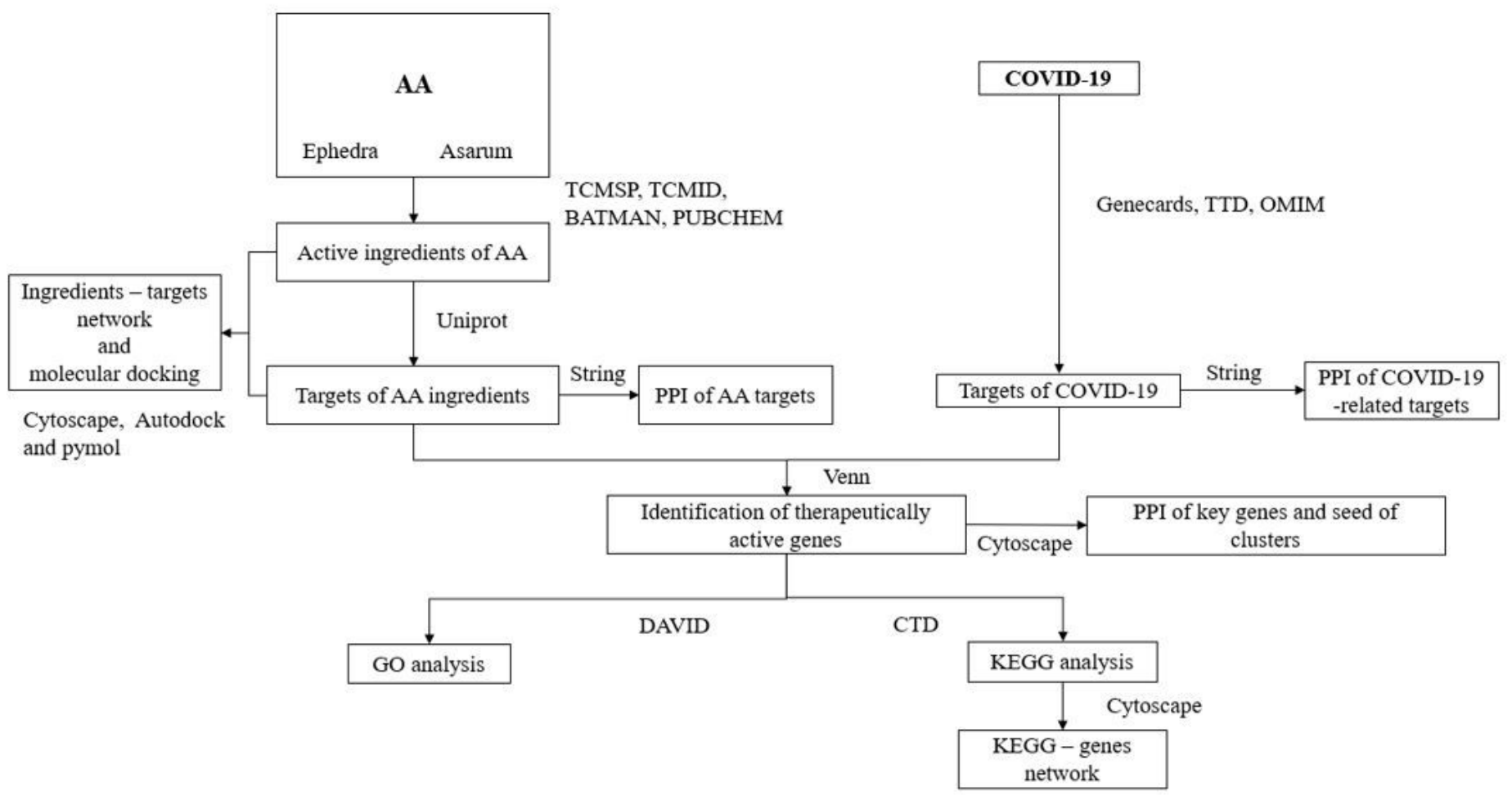

Figure 1

Flowchart of the study methods 
$\mathbf{A}$

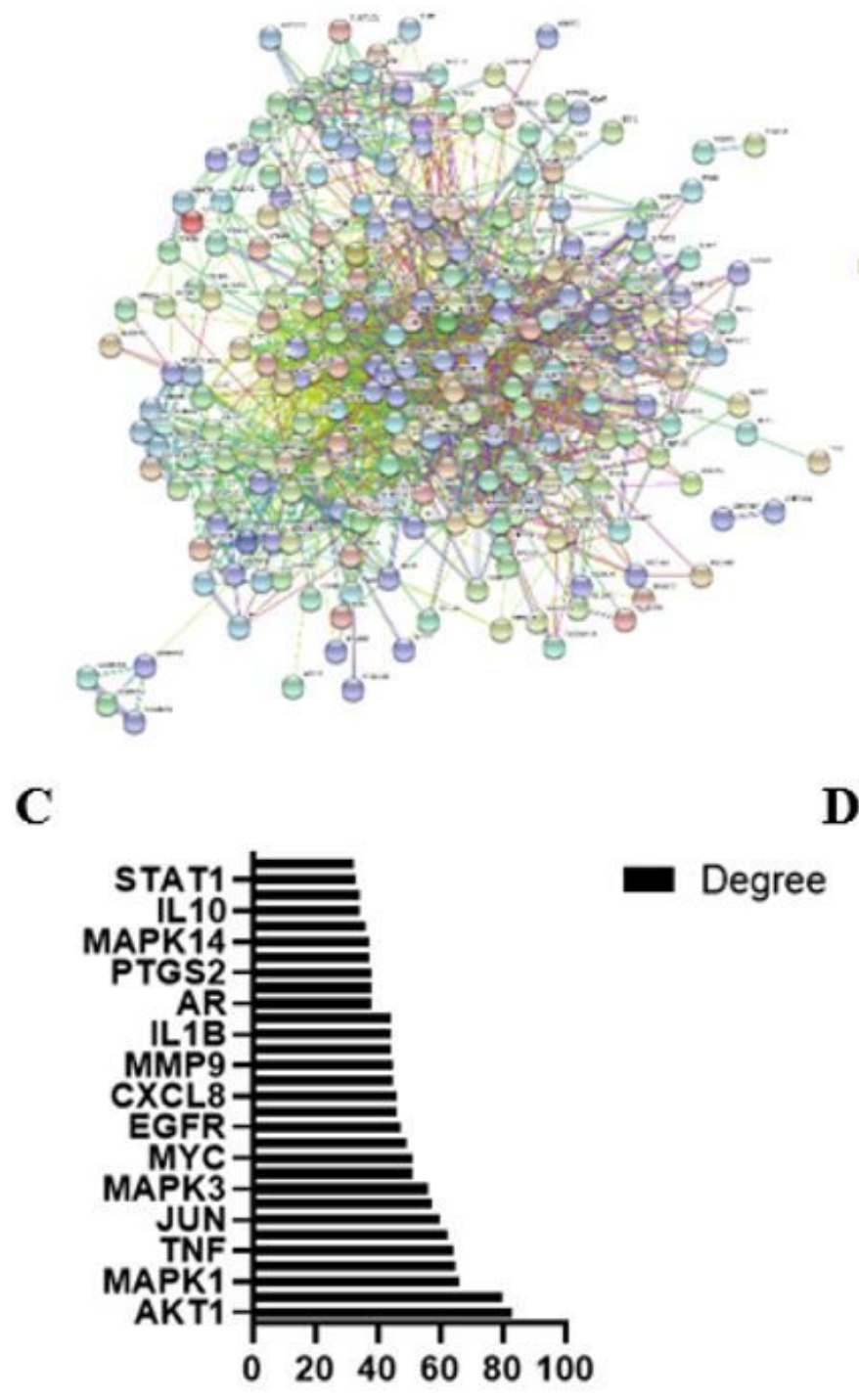

B

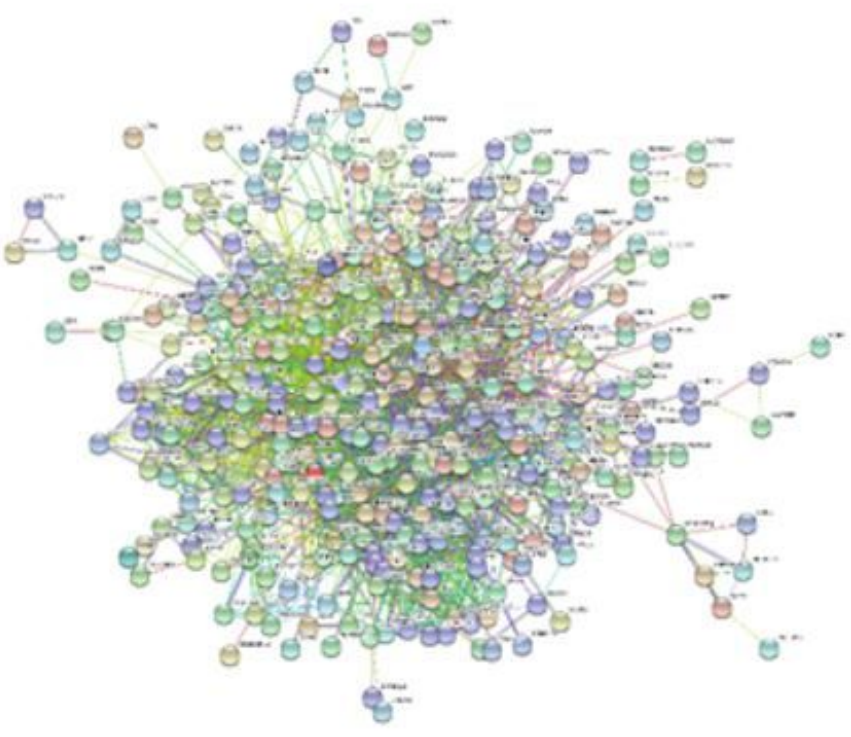

D

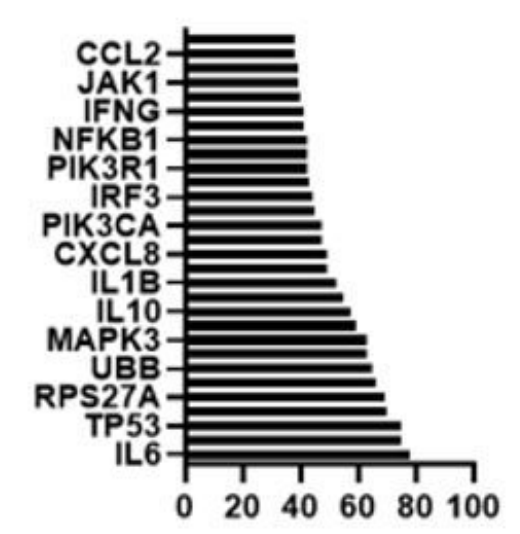

Figure 2

Protein-protein interaction (PPI) network of acupoint application (AA) putative and COVID-19-related target genes and the Barplot of PPI. (A) PPI network of AA putative target genes. (B) PPI network of COVID-19related genes. (C) Barplot showing the significant target genes in the PPI network of AA. (D) Barplot showing the significant target genes in the PPI network of COVID-19. 


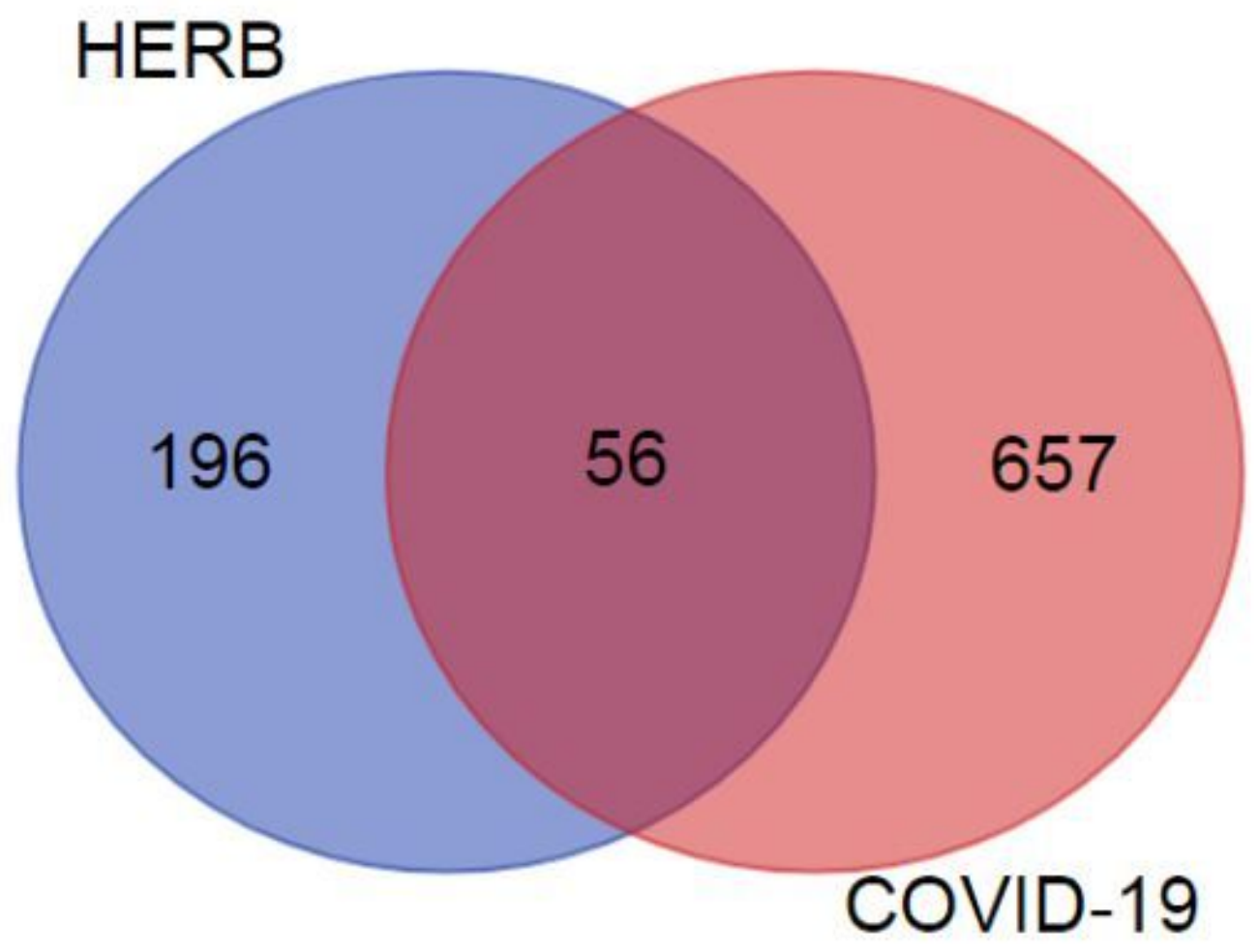

Figure 3

Matching of target genes between COVID-19 and herbs used in acupoint application (AA). 
A

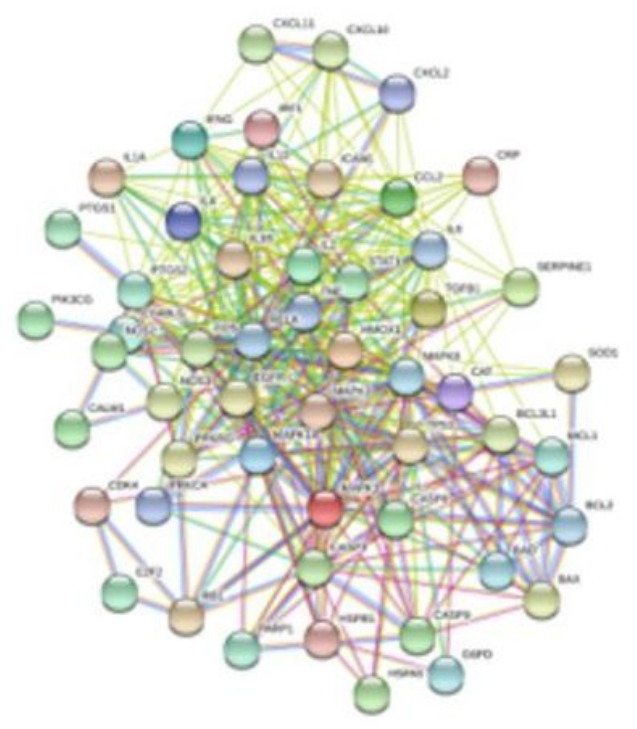

B

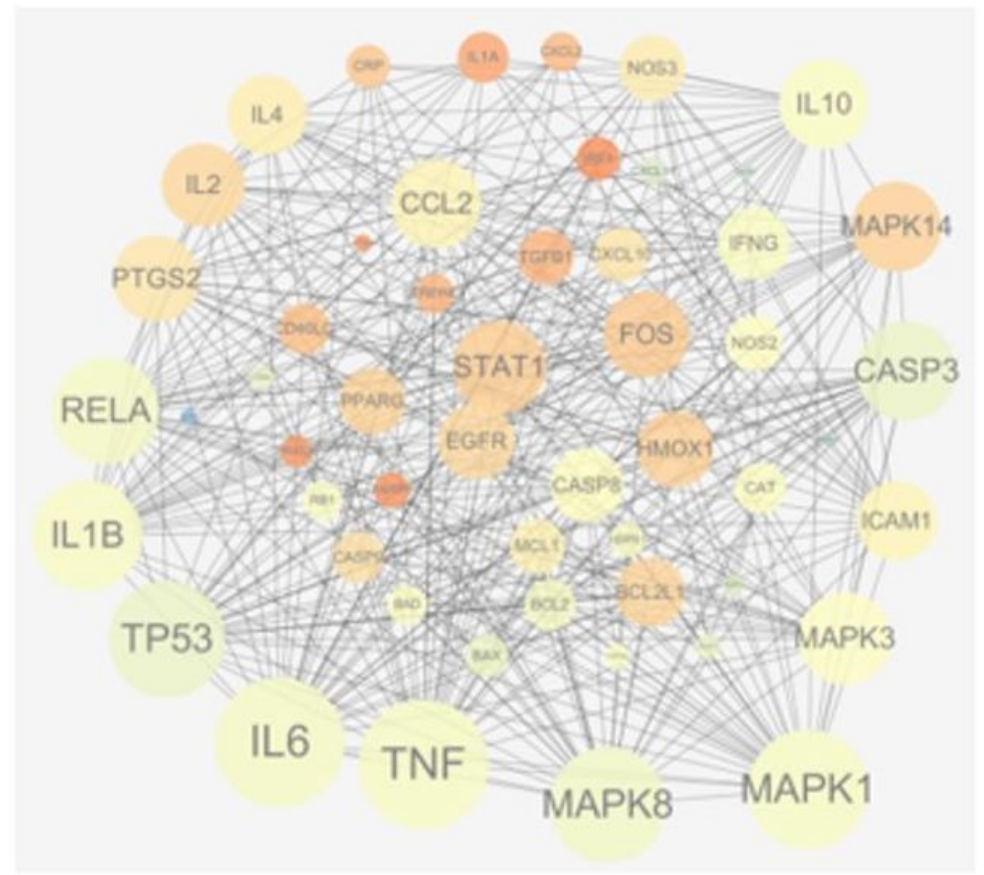

\section{Figure 4}

Protein-protein interaction (PPI) network analysis of acupoint application (AA) against COVID-19. (A) PPI network of the key targets for treating COVID-19. (B) PPI subnetwork of the key targets for treating COVID19. 


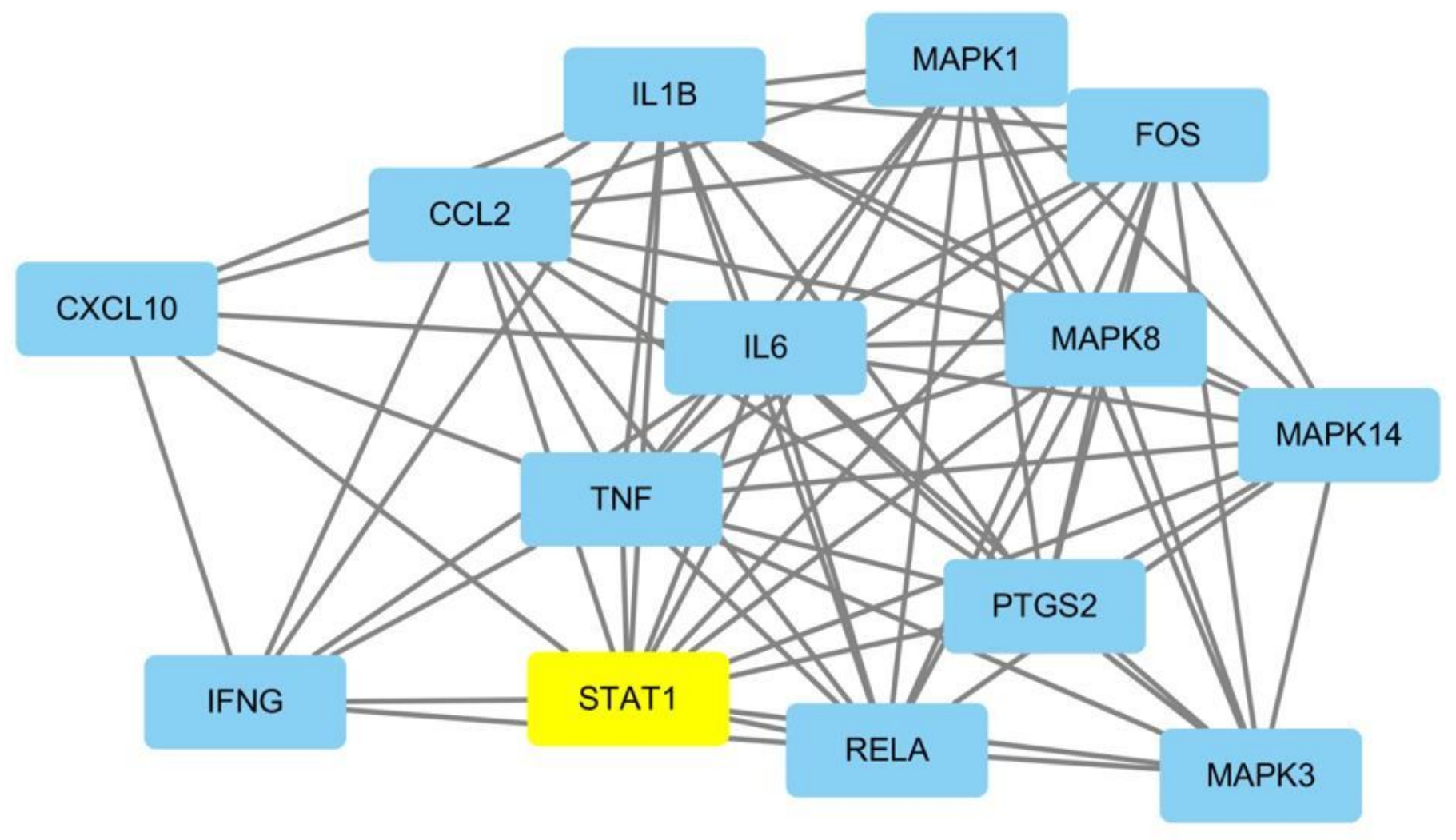

Figure 5

Module cluster with the highest score 


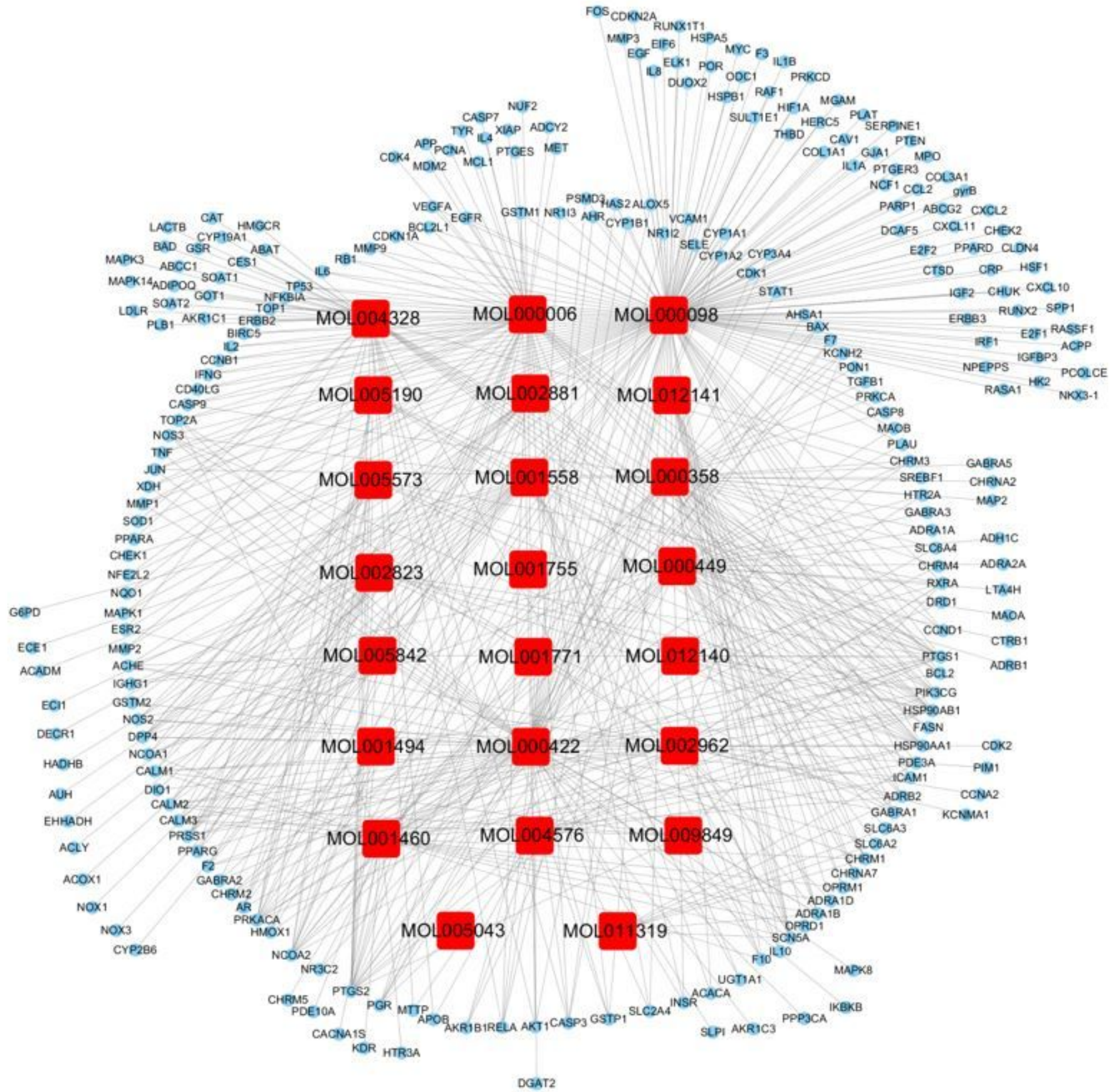

Figure 6

Compound-Target Network 
$\mathbf{A}$

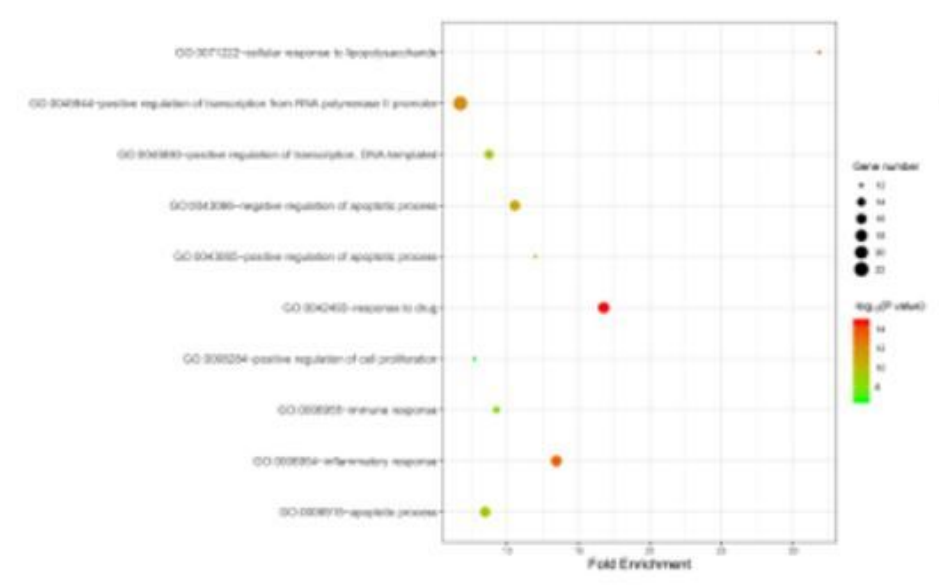

B

$\mathrm{C}$

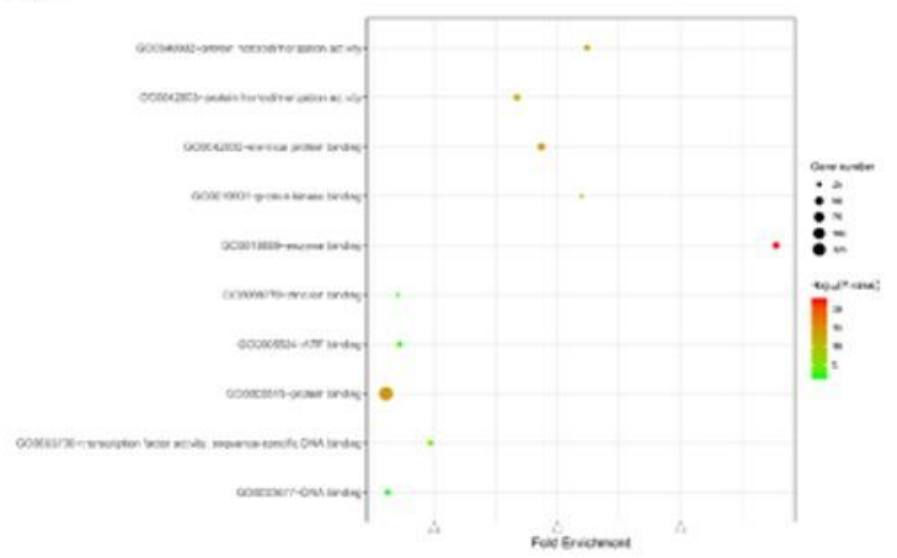

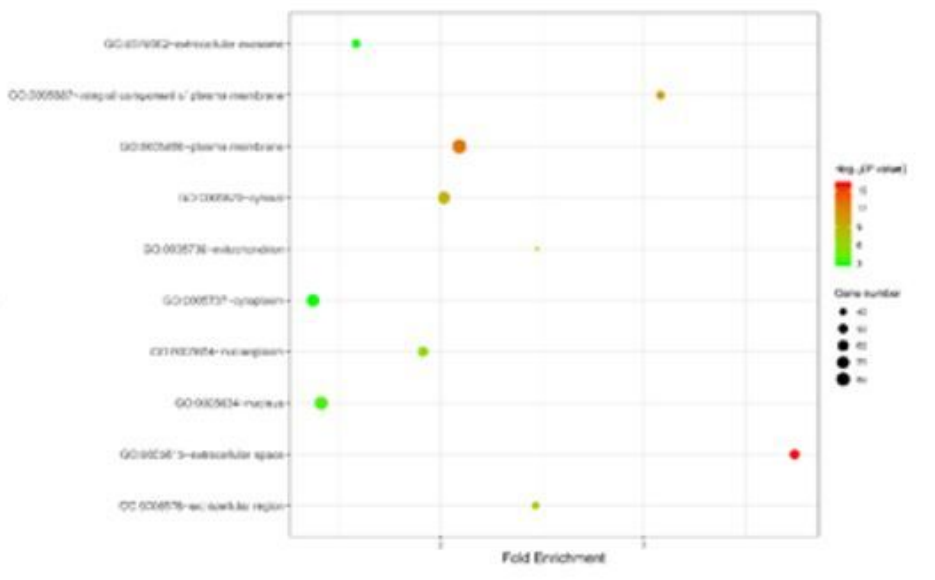

\section{Figure 7}

Gene ontology (GO) enrichment of therapy target genes. (A) BP: biological processes. (B) CC: cellular components. (C) MF: molecular functions. 

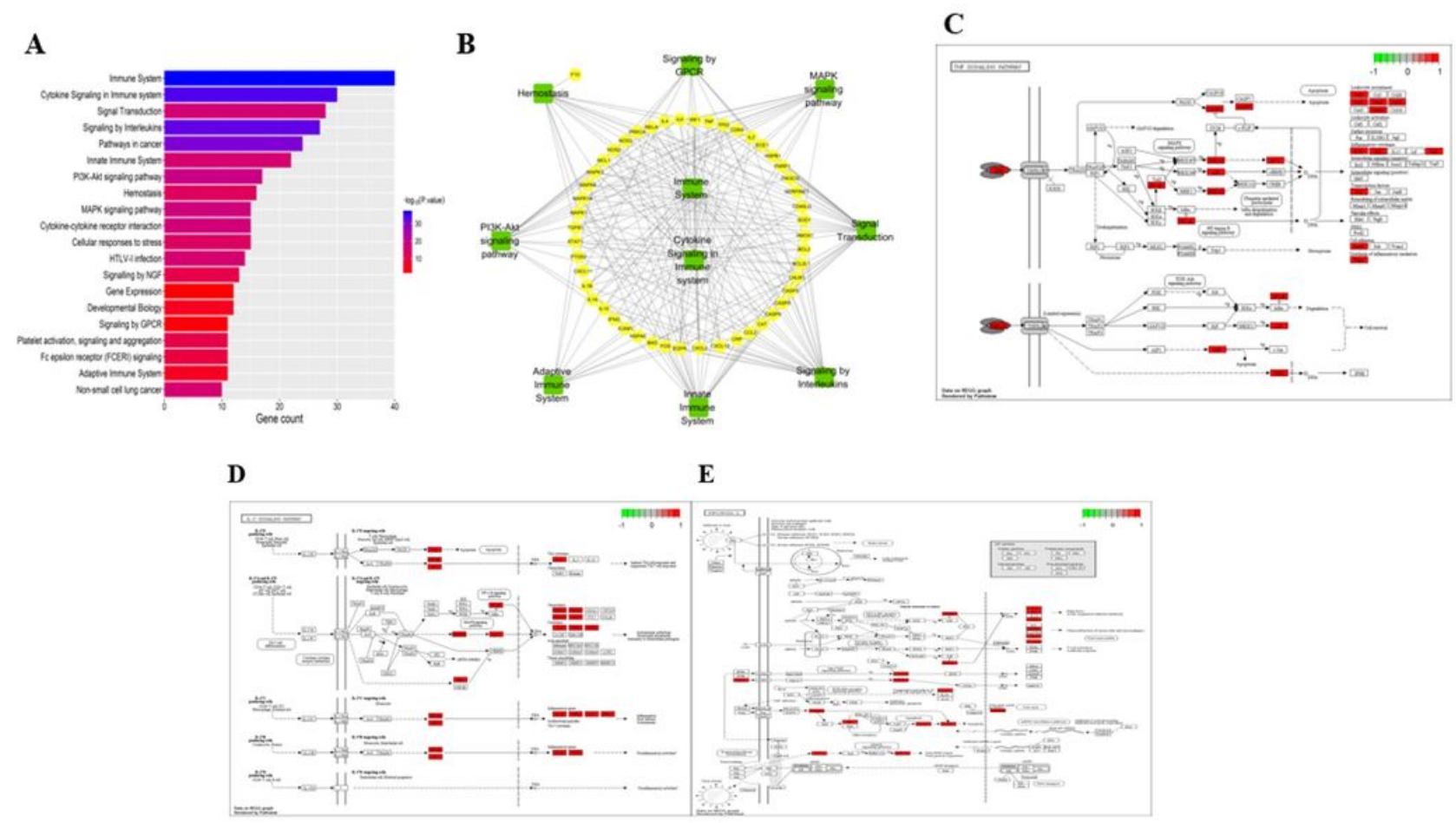

\section{Figure 8}

KEGG pathway enrichment analysis. (A) Twenty related pathways with Corrected $P$ value $\leq 0.01$ and gene frequency $>0.5 \%$. (B) Target-pathway network diagram consisting of 10 pathways with a higher degree value and target genes. (C) Specific mechanism of gene enrichment in the TNF signaling pathway. (D) Specific mechanism of gene enrichment in the IL-17 signaling pathway. (E) Specific mechanism of gene enrichment in the Influenza A signaling pathway. 
A

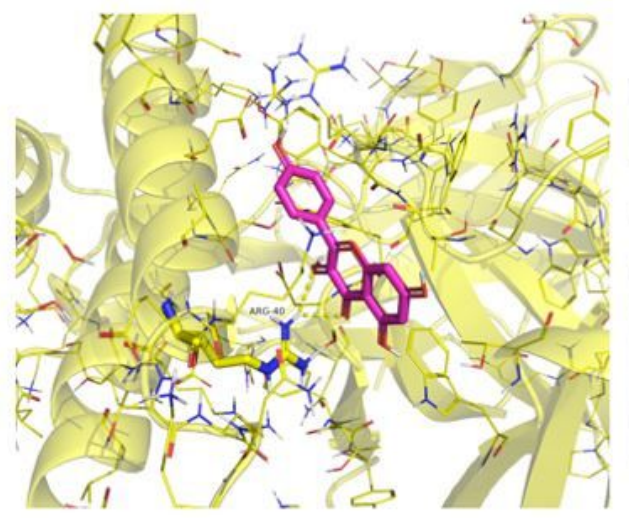

D

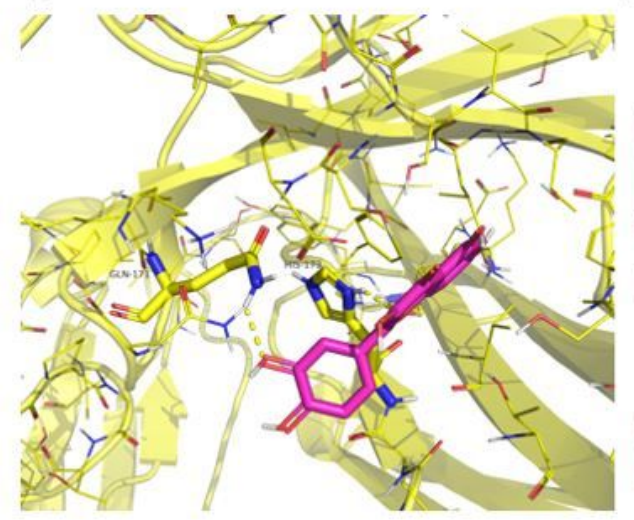

B

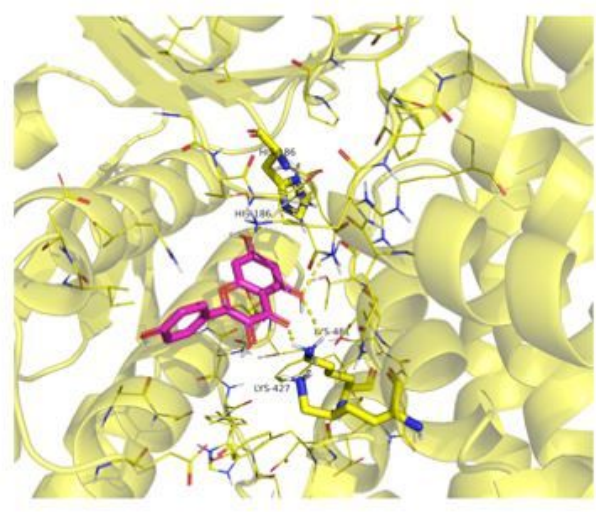

E

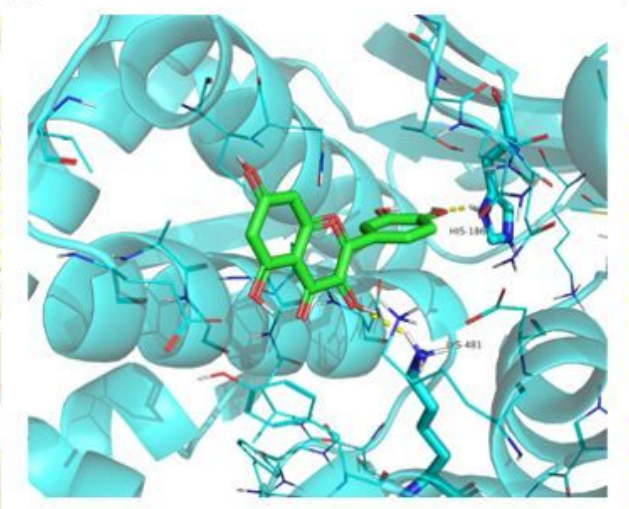

C

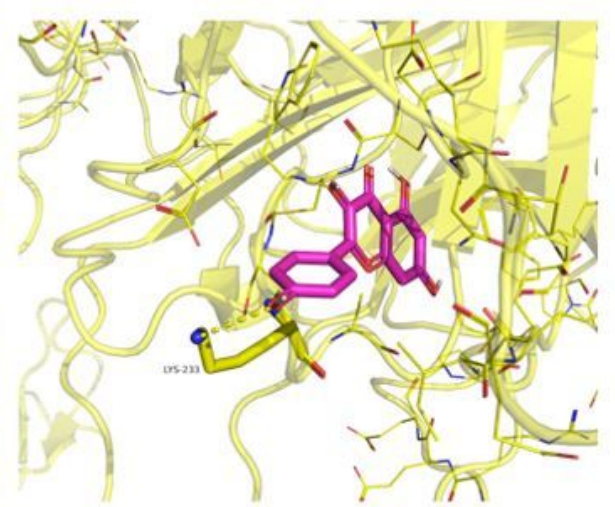

F

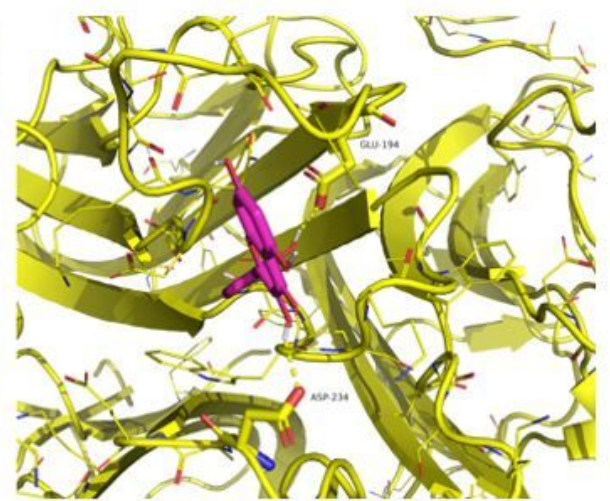

\section{Figure 9}

Molecular docking results. (A) Kaempferol docking with IL-6. (B) Kaempferol docking with STAT1. (C) Kaempferol docking with TNF. (D) Quercetin docking with IL-6. (E) Quercetin docking with STAT1. (F) Quercetin docking with TNF.

\section{Supplementary Files}

This is a list of supplementary files associated with this preprint. Click to download.

- Tables.docx 